\title{
Influence of Binaural Beats on EEG Signal
}

\author{
C. KASPRZAK* \\ Department of Mechanics and Vibroacoustics, Faculty of Mechanical Engineering and Robotics \\ University of Science and Technology, al. A. Mickiewicza 30, 30-059 Kraków, Poland
}

\begin{abstract}
Binaural beats are subjective hearing sensations, which occur when one of tone signals is applied to one ear, and the other one, with a slightly different frequency is applied to the second ear. A listener then receives a resultant sound with an amplitude which changes with a frequency equal to the difference of frequency of applied signals. The aim of this thesis was to examine the influence of binaural beats on changes in the morphology of the brain bioelectrical signal. Verification of previous studies, such as stimulation of binaural beats affects the brain and can cause changes in other frequency bands. Previous studies have been conducted on individual leads and dealt with the occurrence of follow up effect. In the research there were used binaural beats with a frequency of $f=10 \mathrm{~Hz}$. The left ear was exposed to a signal with a frequency of $100 \mathrm{~Hz}$, and the right ear — to a signal with a frequency of $110 \mathrm{~Hz}$, the acoustic pressure level SPL $=73 \mathrm{~dB}$. The research was conducted on a sample group of 20 people. The analysis of average amplitudes of spectral density function of EEG strength signal proved that the exposition of binaural beats brought about a follow-up effect, which means that a component frequency in the EEG signal morphology was observed which corresponds with a frequency of the exposed binaural beats. It was also noted that during the exposition of binaural beats, there occurs a statistically significant decrease of average amplitudes of spectral density function of EEG strength signal for alpha and beta frequency ranges. However, it was observed that the amplitude of spectral density function of the strength has increased in theta frequency range.
\end{abstract}

PACS: $87.50 . Y-$

\section{Introduction}

Binaural beats are subjective hearing sensations, which occur as a result of receiving two slightly different sounds with low frequency. They were discovered in 1839 by a German experimentator H.W. Dove. The ability of a human to hear binaural beats seems to be a result of evolutionary assimilation. Many advanced species can detect binaural beats, depending on a skull size.

Binaural beats are a result of superposition of neuron discharge coming from the left and right ear on a suitable level of the hearing route. Binaural beats prove that neuron discharge in the auditory nerve maintain the information about the phase of a received signal. Due to the fact that such discharges, in accordance with synchronization phenomena, appear for a specified signal phase, the result of this superposition depends on a phase difference occurring between the presented signal, which is a mapping of the binaural difference of their frequency [1-3].

The effect of hearing of sound binaural beats occurs only when we listen to two tonal (sinusoid-like) sounds with almost identical frequencies; yet each of these sounds is heard by one ear only thanks to the use of headphones or stereo speakers. A nerve hearing route in the brain guarantees the exchange of auditory infor-

* e-mail: cekasp@agh.edu.pl mation between the two sides, before the sound reaches the cortical cortex, ensuring its conscious hearing. Such an exchange happens in at least two places of the auditory route - in the olivary body - in auditory centers which process sounds and which receive stimulation directly from the cochlea nucleus and transfer it (each of them to both sides) to colliculus inferior - small nuclei, which constitute the next element of the auditory route, and then through a commissure of the colliculus inferior. Two signals are connected in the brain, the result being a sensation of hearing a third signal — with a frequency of a signal provided to the left and the right ear-called binaural beats $[4,5]$.

Listening to binaural beats provides information to the reticular system. This system is also called a diffuse activating system, which is a big brain area resembling a net. It decides about lucidity, concentration and consciousness. If internal stimuli (feelings, demeanors or beliefs) or external (sensory ones) are not in conflict with this information, then the reticular system changes the brain wave activity so that it is adjusted to the stimulation of the beat signals. It is a natural function of homeostasis. The brain automatically and actively regulates all body functions so that homeostasis is maintained, which is a kind of an inner balance. Trying to maintain homeostasis in a natural way, the reticular system monitors and extorts maintaining sustainable states of cortical cortex of wave activity all the time (unless new piece of information which should be reacted to is delivered - either 
from external sources or from sensory senses). Thanks to the fact that the frequency characteristics of the auditory field are similar to the characteristics of brain waves, the reticular system initiates processing by the cortical cortex the information carried by these signals, believing that the information carried by binaural beats come from the actual brain wave activity. With time, the reticular system monitors the internal and external environment as well as the psychophysical state in order to determine whether there is a need for adjusting to new conditions. Unless there are conflicts, the reticular system continues adjusting the character, quality and features of the consciousness state to the information carried by the signals of binaural beats $[6,7]$.

Owens and Atwater examined EEG patterns associated with listening to a series of low-frequency binaural beats and investigated some of the subjective experiences accompanying such stimulation. In this study, subjects listened through stereo headphones to pure tones designed to produce delta and theta binaural beats. Analysis of the EEG data involved computing the changes in the percentages of total EEG amplitudes, comparing the conditions of waking rest, binaural-beat stimulus periods, and a second period of rest. Results showed that during the stimulus periods subjects generated significantly less alpha- $(p<0.01$ to $p<0.0001)$ and beta- $(p<0.04$ to $p<0.002)$ frequency brain waves and significantly more delta- $(p<0.002$ to $p<0.0009)$ and theta- $(p<0.01$ to $p<0.0001)$ frequency brain waves. This investigation suggested that binaural beats may be associated with reduced EEG arousal. Results of the two other studies showed that during the binaural-beat stimuli, reductions in the percentages of occipital alpha (bipolar O1-O2) were significant (individually, $p<0.05$, and together, $p<0.001$ ) during five of six free-running EEG recording periods compared to baselines. During the same recording periods, reductions in the percentages of central delta (bipolar C3-C4) were similarly significant during four of six periods compared to baselines [8-10].

\section{Methodology of research}

The experiment was conducted on a sample of 20 males. The examined individuals were volunteers, who declared that they had no any medical conditions and not under the influence of medicines. They had been also informed that before the experiment, they were not allowed to drink any stimulating nor intoxicating drinks.

In the research, there were used binaural beats with an acoustic pressure level of SPL $=73 \mathrm{~dB}$, with the following frequencies: the right ear $-110 \mathrm{~Hz}$, the left ear - $100 \mathrm{~Hz}$. The total duration of the experiment was $35 \mathrm{~min}$. The exposition of the stimulus was $20 \mathrm{~min}$. The acoustic signal, recorded in a wave format, was played from a computer onto stereo headphones.

The EEG test was about a registration (with a help of electrodes placed on the skin of a head) of functional currents of a human brain, whose characteristic feature is small tension (from several to several hundred microvolts). The frequency of these currents ranges from $0.5 \mathrm{~Hz}$ to $50 \mathrm{~Hz}$. The registration of EEG signal was conducted with a help of 25-channel sound box of SAM 25 type of MICROMED company.

EEG cap was fitted in accordance with a standard 10/20 system, where electrodes are placed along the sagittal line of the head ( 5 on the left side: Fp1, F3, $\mathrm{C} 3, \mathrm{P} 3, \mathrm{O} 1$ and 5 on the right side: $\mathrm{Fp} 2, \mathrm{~F} 4, \mathrm{C} 4, \mathrm{P} 4, \mathrm{O} 2$ and a reference electrode on the $\mathrm{OP}, \mathrm{Pz}$ ).

The examined individuals were informed about the general target of the research, rules of the experiment and signed a permission form confirming their conscious agreement for the experiment. Later, after having cleaned the skin, measurement electrodes of EEG signals were fitted. After checking the effective resistance (proper applying of the electrodes), a tested person was comfortably seated on a testing site.

After conducting preparation activities, a proper experiment took place; that is $35 \mathrm{~min}$ of constant acquisition of EEG human bioelectric signals. The initial $5 \mathrm{~min}$ was without the binaural beats exposition, $20 \mathrm{~min}$ with the signal exposition and $10 \mathrm{~min}$ - without the exposition.

\section{Result analysis}

The obtained EEG runs were checked regarding the correctness of recording. Two people, due to high disturbances in all the recording were ruled out from EEG analysis. Moreover, there were errors and artifacts noted on some channels for 7 people. The data from these channels were not analyzed.

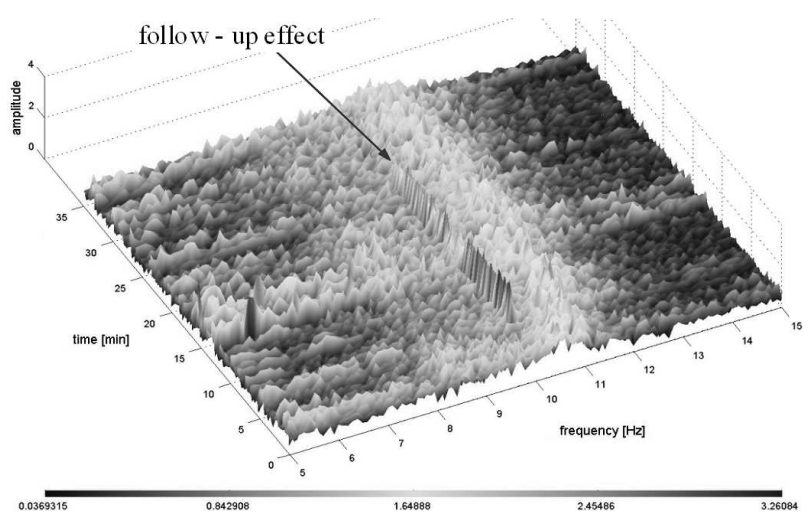

Fig. 1. A follow-up effect.

Basing on the analysis of a spectral density function of EEG strength signal, for 4 people a follow-up effect was observed; i.e. there occurred a component in EEG signal morphology, with a frequency of the presented binaural beats $(10 \mathrm{~Hz})$, Fig. 1.

The changeability of an average amplitude of spectral density function of EEG strength signal in a frequency range from 9.9 to $10.1 \mathrm{~Hz}$ (single channel, C4) was presented in Fig. 2. The plots indicated subsequent stages of 
the study and averages amplitude of the spectral density function of EEG signal in a selected frequency range.

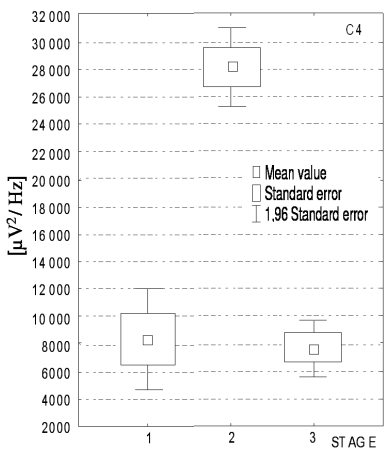

Fig. 2. A diagram of an average amplitude of a spectral density function of EEG strength signal changes in a frequency range from 9.9 to $10.1 \mathrm{~Hz}$.

The above figure confirms the occurrence of the follow-up effect at a previously selected person. The conducted quality analysis (the diagrams of a spectral density function of EEG strength signal changes) were com- pleted with quantity analysis of a spectral density function of EEG strength signal changes in selected frequency ranges. In order to achieve this, there was assumed an EEG signal frequency division used in the electroencephalography:

- beta — from 12.0 to $29.9 \mathrm{~Hz}$

- alpha - from 8.0 to $11.9 \mathrm{~Hz}$,

- theta - from 4.0 to $7.9 \mathrm{~Hz}$,

- delta - from 0.5 to $3.9 \mathrm{~Hz}$.

Using ANOVA test, the statistical significance of differences of an average amplitude of a spectral density function between three consecutive stages of the research is obtained. In Table I there is placed a level of the statistical significance of differences of an average amplitude of a spectral density function in the adopted frequency ranges. In the first column, there are placed analyzed frequency ranges. The following columns correspond to individual measurement EEG channel (from Fp1 to O2).

Statistical significance of differences between consecutive stages of the research

TABLE I for 10 channels in adopted frequency ranges.

\begin{tabular}{c|c|c|c|c|c|c|c|c|c|c}
\hline \hline \multirow{2}{*}{$\begin{array}{c}\text { The analyzed } \\
\text { frequency ranges }\end{array}$} & \multicolumn{10}{|c}{ Statistical significance $p$} \\
\cline { 2 - 11 } & Fp1 & F3 & C3 & P3 & O1 & Fp2 & F4 & C4 & P4 & O2 \\
\hline delta & 0.8295 & 0.1744 & 0.1212 & 0.3469 & 0.1215 & $0.0018^{*}$ & 0.5168 & 0.8197 & 0.1200 & 0.6795 \\
theta & $0.0328^{*}$ & $0.0052^{*}$ & 0.1196 & $0.0006^{*}$ & $0.0010^{*}$ & $0.0000^{*}$ & 0.6406 & $0.0223^{*}$ & $0.0000^{*}$ & 0.2805 \\
alpha & $0.0000^{*}$ & $0.0000^{*}$ & $0.0000^{*}$ & $0.0000^{*}$ & $0.0000^{*}$ & $0.0000^{*}$ & $0.0000^{*}$ & $0.0000^{*}$ & $0.0001^{*}$ & $0.0000^{*}$ \\
beta & $0.0009^{*}$ & $0.0000^{*}$ & $0.0064^{*}$ & $0.0000^{*}$ & $0.0000^{*}$ & $0.0000^{*}$ & $0.0000^{*}$ & $0.0161^{*}$ & $0.0000^{*}$ & 0.2595 \\
\hline
\end{tabular}

The values with star show changes which are statistically significant.

Figures 3, 4, 5 are present changes of average amplitudes of spectral density functions for adopted frequency ranges: theta, alpha and beta (for single channel).

The above figures show an increase of average amplitudes of a spectral density function of EEG strength signal for theta frequency range during the exposition of binaural beats and a falling tendency during stage 3 . In three cases, these changes turned out to be statistically insignificant.

The changes of the average amplitude of a spectral density function of EEG strength signal in three stages of the experiment for alpha frequency range are presented in Figs. 4-7.

In the figures, it can be observed that changes of average amplitudes of a spectral density function of EEG strength signal for alpha frequency range have a falling tendency during the exposition of binaural beats (stage 2), whereas stage 3 , when compared to stage 2 , generates a slight increase. The changes of these values are statistically significant. In the above figures, one can notice that the changes of average amplitudes of a spectral density function of EEG strength signal for alpha frequency range have a falling tendency during the exposition of binaural beats (stage 2 ), whereas stage 3 , when compared to stage 2 , generates a slight increase.

The changes of average amplitudes of a spectral density function of EEG strength signal in three stages of the experiment for beta frequency range are presented in Fig. 5.

In the next stage, all 10 channels (Fp1, F3, C3, P3, O1, $\mathrm{Fp} 2, \mathrm{~F} 4, \mathrm{C} 4, \mathrm{P} 4, \mathrm{O} 2)$ for the adopted frequency ranges were averaged. In Table II there is presented the statistical significance of changes of average amplitudes of a strength function of spectral density for the analyzed variables in consecutive stages of the experiment. 


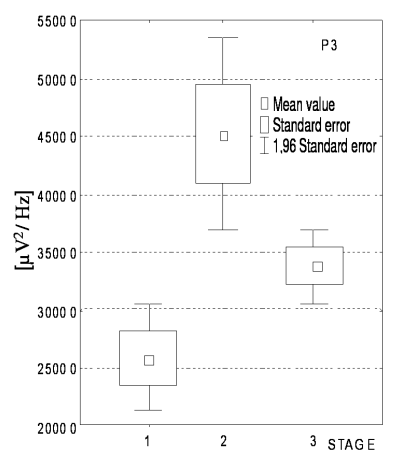

Fig. 3. Analysis of changes of an average amplitude of a spectral density function of EEG strength signal in 3 consecutive stages of the experiment, for theta frequency range (channel P3).

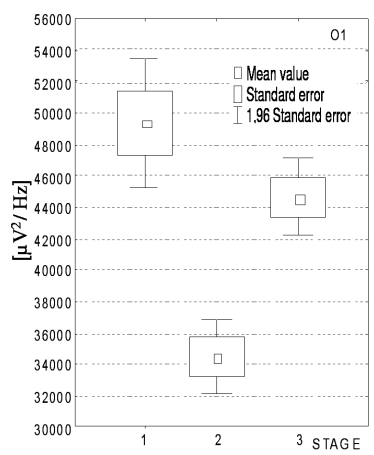

Fig. 4. Analysis of changes of an average amplitude of a spectral density function of EEG strength signal in 3 consecutive stages of the experiment, for alpha frequency range (channel O1).

\section{TABLE II}

Statistical significance of the average value changes from 10 channels, between consecutive stages of the research for individual frequency ranges.

\begin{tabular}{c|c}
\hline \hline $\begin{array}{c}\text { The analyzed } \\
\text { frequency ranges }\end{array}$ & $\begin{array}{c}\text { Statistical } \\
\text { significance } p\end{array}$ \\
\hline delta & 0.4 \\
theta & $0.0231^{*}$ \\
alpha & $0.0000^{*}$ \\
beta & $0.0000^{*}$ \\
\hline
\end{tabular}

The values with star show changes which are statistically significant.

In Figs. 4-7 there are presented changes of average amplitudes of a spectral density function for the analyzed variables (an average value delta, an average value theta, an average value alpha, an average value beta) in three consecutive stages of the experiment.

Figure 6 proves that an average amplitude of a strength function of spectral density for an average value delta increases during the exposition of binaural beats (stage 2),

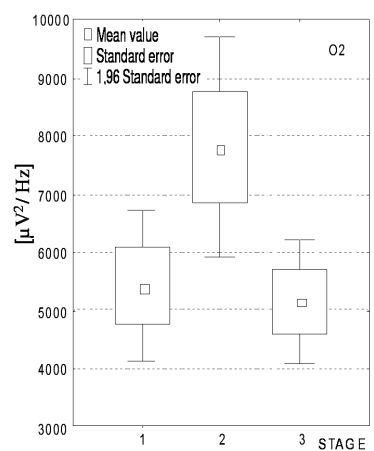

Fig. 5. Analysis of changes of an average amplitude of a spectral density function of EEG strength signal in 3 consecutive stages of the experiment, for beta frequency range (channel O2).
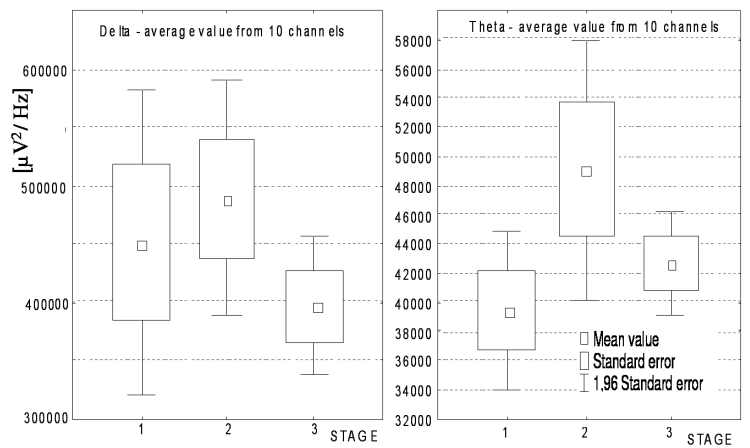

Fig. 6. Analyses of changes of average amplitudes of a spectral density function of EEG strength signal for average values delta and theta (average value from 10 channels).

however there is a decrease of it in stage 3 . Nevertheless, these changes are statistically insignificant. For an average value theta, a similar tendency can be observed, with the difference however, that it is statistically significant.

In both cases of alpha and beta average values, one can observe a decrease of an average amplitude of a strength

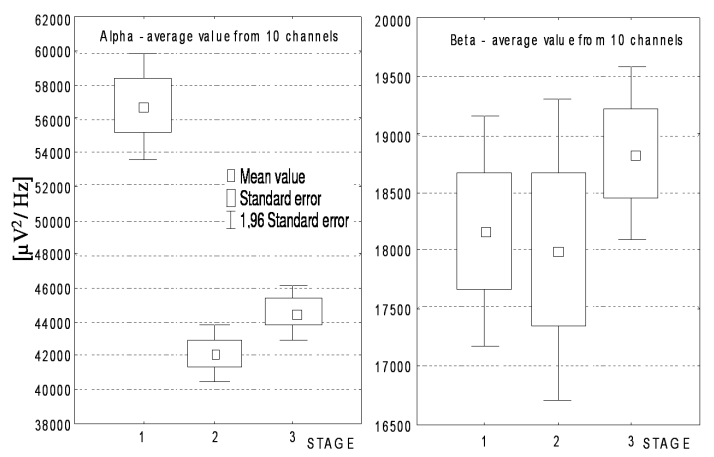

Fig. 7. Analyses of changes of average amplitudes of a spectral density function of EEG strength signal for average values alpha and beta (average value from 10 channels). 
function of spectral density during binaural beats exposition (stage 2) and its increase in stage 3 (when compared to stage 2 ). These changes are statistically significant.

The analyses of changes of average amplitudes of a spectral density function of EEG strength signal proved that the binaural beats exposition brought about a follow-up effect at 4 experiment participants, meaning that there was observed a frequency component in EEG signal morphology, with a frequency corresponding to the presented binaural beats. It was also noted that during binaural beats exposition, there occurs a statistically significant fall of average amplitudes of a spectral density function of EEG strength signal for alpha and beta frequency ranges. However, for theta frequency range, one can observe an increase of average amplitudes of a spectral density function of EEG strength signal. In case of delta frequency ranges, there were no statistically significant changes.

\section{Conclusions}

This study reported and compiled provide statistical observations in support of the notion that binaural beats appear to engender changes in cortical arousal, which can be monitored with the EEG. The results obtained on the basis of the conducted experiment of the influence of binaural beats with $f=10 \mathrm{~Hz}$ frequency and with an acoustic level at SPL $=73 \mathrm{~dB}$ on a human confirm that binaural beats exposition cause statistically significant changes of EEG signal (morphology).

Binaural beats exposition brought about a follow-up effect (in qualitative analysis) in four experiment participants, meaning that there was observed a frequency component in EEG signal morphology, with a frequency corresponding to the presented binaural beats.

It was also noted that during the binaural beats exposition, there occurs a statistically significant fall of average amplitudes of a spectral density function of EEG strength signal for alpha $(p<0.001)$ and beta $(p<0.001)$ frequency ranges. For theta $(p<0.0231)$ frequency range, however, there was noted an increase of a spectral density function of EEG strength signal. In case of delta frequency range, there were no statistically significant changes noted.

The study showed a statistically significant reduction of alpha rhythm $(8-12 \mathrm{~Hz})$ while increasing narrow band share in the range $9.9-10.1 \mathrm{~Hz}$. The reasons may be as follows: blocking of alpha rhythm with simultaneous occurrence of the phenomenon of follow up effect. This may be due to the orienting reflex nervous system, i.e. a response to the acoustic stimulus and tuning to optimize the nervous system receiveing information from the environment, which manifests itself, inter alia, by blocking the alpha rhythm. However, observed increase in amplitude for a frequency corresponding to the binaural beat frequency $f=10 \mathrm{~Hz}$ based on the assumption that the human brain has a tendency to change its dominant EEG frequency towards the frequency of a dominant external stimulus.

\section{Acknowledgments}

This study is a part of the N N501 247740 research project, supported by the National Science Centre.

\section{References}

[1] E. Ozimek, Sound and Its Perception. Aspects of Physical and Psychoacoustics, PWN, Warszawa 2002.

[2] C. Kasprzak, Acta Phys. Pol. A 118, 87 (2010).

[3] C. Kasprzak, Acta BioOptica Inform. Med. 15, 390 (2009).

[4] C. Kasprzak, Pol. J. Environmental Studies 16, 217 (2007).

[5] R. Padmanabhan, A.J. Hildreth, D. Laws, Anaesthesia 60, 874 (2005).

[6] J. Smith, J.J. Marsh, W.S. Brown, Electroencephalography Clinical Neurophysiology 39, 465 (1975).

[7] H. Wahbeh, C. Calabrese, H. Zwickey, J. Alternat. Complement. Med. 13, 25 (2007).

[8] F. Atwater, Monroe Institute J. 1, 1 (2009).

[9] J. Lane, S. Kasian, J. Owens, Physiol. Behav. 63, 249 (1998).

[10] P. Petty, J. Consciousness Studies 5, 86 (1998). 